OPEN ACCESS

Edited by:

Hiroshi Yamada

University of Tsukuba, Japan

Reviewed by:

Veit Stuphorn,

Johns Hopkins University,

United States

NaYoung So,

Columbia University, United States

*Correspondence:

Satoshi Nonomura

satoshi.nonomura@gmail.com

Specialty section:

This article was submitted to

Decision Neuroscience,

a section of the journal

Frontiers in Neuroscience

Received: 28 March 2019 Accepted: 12 November 2019 Published: 28 November 2019

Citation:

Nonomura S and Samejima $K$ (2019) Neuronal Representation of Object Choice in the Striatum of the

Monkey. Front. Neurosci. 13:1283. doi: 10.3389/fnins.2019.01283

\section{Neuronal Representation of Object Choice in the Striatum of the Monkey}

\author{
Satoshi Nonomura ${ }^{1,2 *}$ and Kazuyuki Samejima ${ }^{1}$ \\ 'Brain Science Institute, Tamagawa University, Tokyo, Japan, ${ }^{2}$ Physiology and Cell Biology, Graduate School of Medical \\ and Dental Sciences, Tokyo Medical and Dental University, Tokyo, Japan
}

According to a widely held view, the decision-making process can be conceptualized as a two-step process: "object choice," which does not include physical actions, followed by "movement choice," in which action is executed to obtain the object. Accumulating evidence in the field of decision neuroscience suggests that the cortico-basal ganglia circuits play a crucial role in decision-making. However, the underlying mechanisms of the object and movement choices remain poorly understood, mainly because the two processes occur simultaneously in most experiments. In this study, to uncover the neuronal basis of object choice in the striatum, the main input site of the basal ganglia, we designed a behavioral task in which the processes of object and movement choice were temporally separated, and recorded the single-unit activity of phasically active neurons (PANs) $(n=375)$ in the striatum of two monkeys. We focused our study mainly on neuronal representation during the object choice period, before movement choice, using a mutual information analysis. Population striatal activities significantly represented the information of the chosen object during the object choice period, which indicated that the monkeys actually made the object choice during the task. For the activity of each individual neuron during the object choice period, we identified offered objectand chosen object-type neurons, corresponding to pre- and post-decision signals, respectively. We also found the movement-type neurons during the movement period after the object choice. Most offered object- or chosen object-type neurons were not overlapped with movement-type neurons. The presence of object choice-related signals independent of movement signal in the striatum indicated that the striatum was part of the site where object choice was made within a cortico-basal ganglia circuit.

\section{Keywords: decision-making, object choice, striatum, monkey, electrophysiology, mutual information analysis}

\section{INTRODUCTION}

We often make decisions among abstract outcomes without undertaking physical actions. For example, imagine that you are in a kaitenzushi restaurant (also known as conveyor-belt sushi or sushi train). You can decide on the sushi topping before reaching your hand toward the sushi on the dish carried by conveyor belt. In this case, the first step, which does not include the physical action (reaching your hand), could be regarded as the "object choice"; it is followed by the second step, the "movement choice," in which an action is executed to obtain the object when the object is conveyed in front of you. Recently, several neuroscientists have discussed the concept and neuronal mechanism of the consecutive two-step decision processes (Samejima and Doya, 2007; Padoa-Schioppa, 2011; Cisek, 2012; Chen and Stuphorn, 2015). 
The striatum, the main entry nucleus of the basal ganglia, is thought to play major roles in decision-making. Anatomically, the striatum has inputs from various cerebral cortical areas, including the prefrontal, higher-order motor, and primary motor cortex, and it returns these inputs to the cortical areas largely in parallel via the thalamus (Yeterian and Van Hoesen, 1978; Selemon and Goldman-Rakic, 1985; Alexander et al., 1986; Flaherty and Graybiel, 1993; Haber and Knutson, 2010). Clinically, patients with Parkinson's disease, Huntington's disease, or obsessive-compulsive disorders, all of which are considered disorders of the basal ganglia, exhibit cognitive dysfunction in action choice as well as motor behaviors (Graybiel and Rauch, 2000; Mink, 2003; Frank et al., 2004; Beste et al., 2008). Several lines of evidence from primate and rodent electrophysiological and optogenetic studies have shown that the striatum plays important roles in decision-making by predicting future goals, taking action, and monitoring performance and outcome in order to improve future behavior (Lauwereyns et al., 2002; Takikawa et al., 2002; Cromwell and Schultz, 2003; Samejima et al., 2005; Yamada et al., 2007; Lau and Glimcher, 2008; Cai et al., 2011; Tai et al., 2012; Nonomura et al., 2018).

Note that although there is considerable evidence for the neural basis of decision-making in the striatum, it remains unknown whether and how this region of the brain is involved in the consecutive two-step choice process, i.e., object and movement choice. Because most studies in primates and rodents adopted behavioral tasks in which the alternatives for choice included both motor and non-motor factors simultaneously, e.g., alternatives predicting different reward values (non-motor factor) and the direction of a moving joystick (motor factor) (Samejima et al., 2005), neuronal activity in relation to the object and movement choices could not be clearly dissociated. Several studies have reported that an object signal unrelated to movement direction to guide the choice was represented in the orbitofrontal cortex (OFC), the supplementary eye field (SEF), and the amygdala (Padoa-Schioppa and Assad, 2006; So and Stuphorn, 2010; Grabenhorst et al., 2012; Cai and PadoaSchioppa, 2014; Chen and Stuphorn, 2015). However, few studies have investigated the neuronal representation related to object choice in the striatum.

In this study, to investigate the neuronal representation of object choice in the striatum, we designed a choice task, in which consecutive two-step choice processes were temporally decomposed, recorded single-unit activity in the striatum of macaques performing the task, and performed a mutual information analysis. This is the first study to provide an evidentiary neuronal representation of the striatum for object choice.

\section{MATERIALS AND METHODS}

\section{Animals and Surgery}

All experiments were approved by the Animal Research Ethics Committee of Tamagawa University (animal experiment protocol H21/27-14) and were carried out in accordance with the Fundamental Guidelines for Proper Conduct of Animal
Experiments and Related Activities in Academic Research Institutions (Ministry of Education, Culture, Sports, Science and Technology of Japan) and the Guidelines for Animal Experimentation in Neuroscience (Japan Neuroscience Society). All surgical procedures were performed under appropriate anesthesia, and all efforts were made to minimize suffering (see below). Our procedures for primate animal experiments were established in previous studies at Tamagawa University (Nakayama et al., 2008; Yamagata et al., 2009; Hashimoto et al., 2010; Saga et al., 2011; Arimura et al., 2013).

We used two monkeys (Macaca fuscata): monkey 1 (8.5 kg) and monkey $2(8.0 \mathrm{~kg})$. During the experimental sessions, each monkey sat in a chair with its head and both arms restrained and its right wrist left free to enable it to push a button with its hand; the button was installed in front of the chair at waist level. A 19-inch video monitor screen equipped with a speaker to provide sound stimulation was placed in front of the monkey. Eye positions were monitored at $240 \mathrm{~Hz}$ with an infrared eye-tracking system (resolution, $0.25^{\circ}$ in visual angle; EYETRAC6000, Applied Science Laboratories). The distance between the screen and the monkey's eyes was $340 \mathrm{~mm}$. A tube was located near the monkey's mouth to give a reward of apple juice. The amount of reward was controlled by opening and closing an electromagnetic valve via a control signal from a TEMPO system (Reflective Computing, Olympia, WA, United States), which was also used to control the behavioral task, visual stimulus presentation by the liquid crystal display, and the sound stimulus predicting the amount of reward. The order of presentation of the visual stimuli was controlled by custom MATLAB code (Math Works).

\section{Behavioral Task}

Two tasks were designed, a free-choice task and an instruction task. While seated in the chair, the monkey performed the task by operating a push-button with its right hand according to a visual stimulus presenting the alternatives for choice. If the monkey successfully performed the task, an apple juice reward following the reinforcement sound was given. Four different amounts of reward were used (reward 1, $0.095 \mathrm{ml}$; reward 2, $0.190 \mathrm{ml}$; reward 3, $0.284 \mathrm{ml}$; and reward 4, $0.376 \mathrm{ml}$ ). A reinforcement sound corresponding to the amount of reward was repeated before actual delivery of the reward (one to four repetitions of a short, high tone, corresponding to one to four units of reward).

In the free-choice task (Figure 1A), the monkey had to choose one of two objects presented on the screen. Pushing the button located near the monkey's hand started the task, after which a fixation point $(4.5 \times 4.5 \mathrm{~mm}$ white square dot $)$ appeared in the center of the screen. If the monkey maintained its gaze on the fixation point under 1 degree for $0.8 \mathrm{~s}$, a choice cue was presented in a $40 \times 40 \mathrm{~mm}$ square area (under 6.7 degrees) for $0.8 \mathrm{~s}$. The choice cue consisted of two types of objects located at four corners (upper left and right, lower right and left). Each object was $20 \mathrm{~mm}$ in diameter. The choice cues were randomly picked from 16 objects (four colors $\times$ four shapes). After a delay period (0.8-1.2 s), two objects were individually presented again in random order as the first and second target. The monkey had to choose one of the two targets by releasing the button during presentation of the target. If the monkey released the button 
A
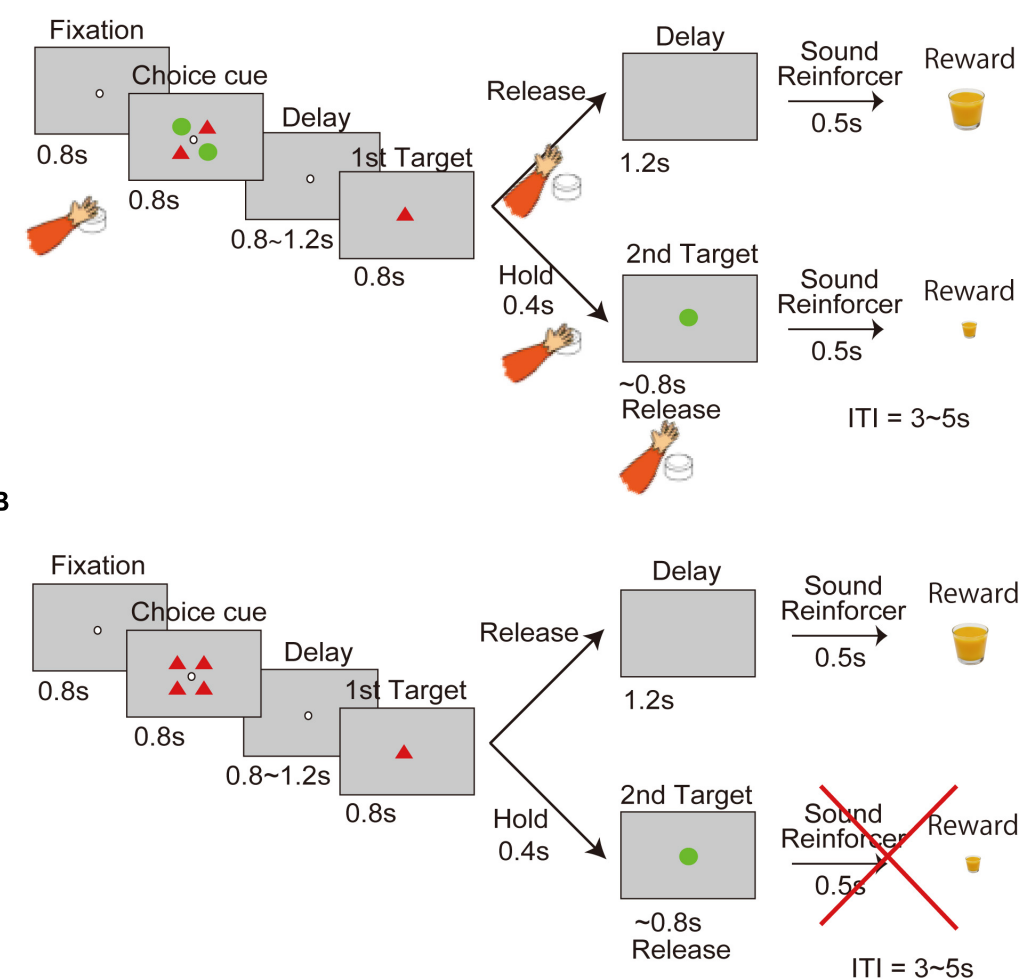

C
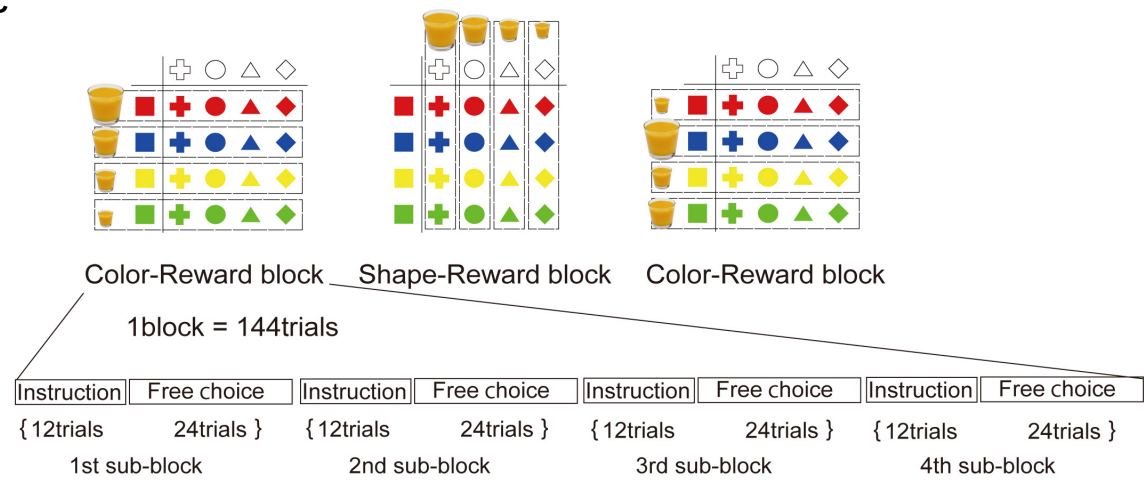

FIGURE 1 | Behavioral task in which the processes of the object and movement choices are temporally dissociated. (A) Free-choice task. Two objects associated with different amounts of reward, without movement factors, were presented as the choice cue for release of the button at a later time. (B) Instruction task. An object was presented as an instruction cue instead of the choice cue in the free-choice task. (C) Object-reward association schedule. The association between four different amounts of reward and object of four different colors and shapes was randomly changed in each block.

during the first presentation of the target $(0.8 \mathrm{~s})$, it received a reward of a size corresponding to the first target after a 1.2-s firstrelease delay period and a 0.5 -s reinforcement sound. Conversely, if the monkey kept holding the button throughout the first target presentation $(0.8 \mathrm{~s})$, the second target was presented following a delay period of $0.4 \mathrm{~s}$. If the monkey released the button during presentation of the second target $(0.8 \mathrm{~s})$, it received a reward of a size corresponding to the second target after a 0.5 $s$ reinforcement sound. Trials were separated by an interval of 3-5 s. A trial was aborted if the monkey failed to maintain fixation of its gaze (over 1 degree) throughout presentation of the fixation point $(0.8 \mathrm{~s})$. When an aborted trial was detected, all presented objects were immediately extinguished, neither the reinforcement sound nor the reward was delivered, and the trial began again.

In the instruction task (Figure 1B), the monkey had to choose only one instructed object presented on the screen. The task sequence was the same as in the choice task, except that in this case, the choice cue was the instruction cue (only one type object).

According to the reward schedule (Figure 1C), the task was run in a block of 144 trials consisting of the first through the fourth subblocks. Each subblock included 12 trials of the instruction task and 24 trials of the free-choice task. Four different amounts of juice reward were associated with four colors or shapes in a block of trials. The association of color with reward 
and shape with reward was altered block by block. The amount of reward associated with each shape or color in a block was randomly changed across every block.

\section{Electrophysiological Recording}

After completing the behavioral training, the monkeys underwent aseptic surgery performed under pentobarbital sodium anesthesia $(20-25 \mathrm{mg} / \mathrm{kg}$, i.v.) with atropine sulfate. Antibiotics and analgesics were used to prevent postsurgical infection and pain. Polycarbonate screws were implanted in the skull, and two plastic pipes, rigidly attached with acrylic resin, were used to securely fix the head during the daily recording sessions. Part of the skull was removed over the anterior part of the striatum, and a recording chamber was implanted, tilted laterally by $35^{\circ}$. To confirm that the chamber was located appropriately to approach the target brain area, magnetic resonance images were recorded. Neuronal activity was recorded with glass-insulated tungsten electrodes (1.0-2 M $\Omega$ at $1 \mathrm{kHz}$; Alpha Omega Engineering) advanced by an oil-driven micromanipulator (MO-97-S; Narishige). The recording sites were determined using a grid system, which allowed us to record at intervals of $1 \mathrm{~mm}$ between penetrations. The electrode was introduced into the brain through a stainless steel guide tube, which was inserted into one of the grid holes and then into the brain through the dura mater. Detection of electrical signals from the electrode and online sorting were performed by a Multichannel Acquisition Processor (MAP/16, Plexon). The signal was amplified by a head-stage (HST/8o50-G20) and pre-amp with a band-pass filter (PBX2/16wb-G50, Plexon; final gain, 500; band-pass filter $0.1-8 \mathrm{kHz}$ ) and collected at $1 \mathrm{kHz}$. The behavioral task was controlled by a TEMPO system and MATLAB. The signals controlling the behavioral task from the TEMPO system were recorded in the MAP system with the neuronal signals. Offline sorting of action potentials was performed with an Offline Sorter (ver3, Plexon). The sorted action potentials and behavioral data were analyzed by MATLAB.

The recording site was the striatum of the left hemisphere (A: $21-30 \mathrm{~mm}$ and L: $18-27 \mathrm{~mm}$ for monkey $1 ; \mathrm{A}: 22-30 \mathrm{~mm}$ and L: $18-28 \mathrm{~mm}$ for monkey 2). The dorsal border of the striatum was easily identified from changes in the background firing rate as the electrode was introduced through the cortex, white matter, and striatum. We classified striatum neurons as phasically active neurons (PANs) or tonically active neurons based on differences in spontaneous activity and spike waveform (Hikosaka et al., 1989; Aosaki et al., 1994). If we judged a PAN to be responsive to any task event by observing a phasic response during a trial, we started recording. All neurons in the database were recorded across at least two blocks of trials, including one shape-reward and one color-reward block.

\section{Data Analysis}

To determine whether the monkey actually made an object choice during the choice cue or delay period, we adopted the latter half of the trials in the block (third and fourth subblocks) for analysis to eliminate the effect of learning about the association between reward and objects. Unless otherwise noted, we analyzed the neuronal data in the free-choice task not including the instruction task. To investigate the neuronal representation related to object choice, we performed mutual information analysis for each recorded neuron (Optican and Richmond, 1987). Mutual information for each neuron was calculated based on the difference between a priori information of a task condition and information of the task condition given the firing rate in the trial. The following equation was used:

$$
\begin{aligned}
I(S ; R) & =H(S)-H(S \mid R) \\
& =\sum_{s}-p(s) \log p(s)-\left\langle\sum_{s}-p(s \mid r) \log p(s \mid r)\right\rangle_{r}
\end{aligned}
$$

where $S$ is the set of task conditions $\{\mathrm{S} 1, \mathrm{~S} 2 \ldots\}, R$ is the set of observed neuronal activities ri: the firing rate in the i-th trial, $H(S)$ is a prior information entropy of the task condition $S, H(S \mid$ $R$ ) is the information entropy of task condition $S$ given neuronal activity $R$ in the trial, and \langle\rangle$_{r}$ is the mean information entropy across all task conditions $s$ given neuronal activity $r$. Here two task conditions $S$ ( $S 1$ and $S 2$ ) were used. The first task condition, S1, was six combinations of the choice cue (referred to as "offered object"), including six color or shape combinations $\left(S 1_{\text {color }}=\right.$ \{red/blue, red/yellow, red/green, blue/yellow, blue/green, and yellow/green $\}$ and $S 1_{\text {shape }}=\{$ circle/triangle, circle/square, circle/cross, triangle/square, triangle/cross, and square/cross $\})$. Under the first task condition, $S 1, p(s)$ was calculated using the probability of $1 / 6$, and $p(s \mid r)$ was calculated using the probability that trials $s$ exhibit higher firing rates than the median firing rate across all trials. The second task condition, $S 2$, was four colors or shapes of the chosen object $\left(S 2_{\text {color }}=\{\mathrm{red}\right.$, blue, yellow, and green $\}$ and $S 2_{\text {shape }}=\{$ circle, triangle, square, and cross\}). Under the second task condition, $S 2, p(s)$ was calculated using the probability of $1 / 4$, and $p(s \mid r)$ was calculated using the probability that trials $s$ exhibit higher firing rates than the median firing rate across all trials.

To find evidence that the monkeys actually made an object choice before a movement choice, we calculated mutual information of the chosen object during the period from onset of choice cue to onset of the first target. Because there was the potential that mutual information of the chosen object had a spurious correlation with that of the offered object, we checked whether the mutual information of the chosen object was significantly larger than that of the information expected from the offered object. To do this, we adopted the bootstrap method for the hypothesis test and calculated the surrogate mutual information of the chosen object in which the information of the chosen object was randomized but the information of the offered object was kept. For example, to calculate the surrogate mutual information of the chosen shape for every recorded neuron, we generated trial-shuffled data in which the chosen shapes were shuffled randomly in every trial group in which the same shape combination of the offered object was presented (irrespective of their colors). We used the trial-shuffled data and calculated the surrogate mutual information of the chosen shape for every recorded neuron. Then, we calculated the summation of surrogate mutual information of the chosen shape from all recorded neurons. We performed this procedure repeatedly 
(10,000 shuffles) and generated the surrogate distribution of the mutual information of chosen shape. The significance level was determined at the top $5 \%$ of the surrogate distribution. If the summation of real mutual information of the chosen shape was more than the significance level in the surrogate distribution, we considered that the real information of the chosen shape was significantly larger than that of the information expected from the offered shape at the population level $(p<0.05)$. In the case of information of the chosen color, we performed the same analysis using color information instead of shape. The dynamics of the summation of real information (Figure 3B) was calculated in 0.2 -s sliding windows with 0.05 steps. The bootstrap method was performed for eight consecutive 0.2-s windows starting from onset of choice cue $0-0.2-\mathrm{s}, 0.2-0.4-\mathrm{s}$, $0.4-0.6-\mathrm{s}, 0.6-0.8$-s, $0.8-1.0-\mathrm{s}, 1.0-1.2-\mathrm{s}, 1.2-1.4$-s, and 1.4-1.6$s$, corresponding to the upper triangles in Figure 3B. As with the chosen object, the significance of mutual information of the offered object was tested ( $p<0.05$ ) (Figure 3C). The statistical test was the same as for the chosen object except that the surrogate mutual information of the offered object was calculated by trialshuffled data in which the offered objects were shuffled randomly in every trial group in which the same object was chosen, e.g., when the chosen object was red, the offered objects (red/blue, $\mathrm{red} /$ yellow, and red/green) were randomized.

To compare two surrogate distributions in Figure 4 by receiver operating characteristic (ROC) analysis for the classification of chosen (or offered) object and value, we recalculated the two surrogate distributions and the area under the curve (AUC) ten times, and compared the AUCs with 0.5 by Mann-Whitney $U$-test.

We also checked the significance of mutual information of the chosen object and the offered object at the single-neuron level (Figure 5). In this case, we compiled the surrogate distribution of mutual information (100 shuffles) in four consecutive 0.4-s windows from choice cue onset for an individual neuron, and then checked whether its real mutual information was larger than the significance level (top 5\% of the distribution of surrogate mutual information, $p<0.05$ ). Furthermore, we performed oneway analysis of variance (ANOVA) with factors of chosen object (color and shape) or offered object (color and shape) for four consecutive 0.4 -s windows $(p<0.05)$. If both statistical tests were passed in the same window, we defined the neuron as the offered object-type (color or shape) or the chosen object type (color or shape).

In addition to the chosen and offered object, we calculated another mutual information using the task condition of "chosen movement" (first or second release) (Figure 6B). To find movement-type neurons in Figures 6A,C,D, we performed oneway ANOVA with factor of chosen movement in the 0.8-s window from onset of the first target $(p<0.05)$.

\section{RESULTS}

\section{Behavioral Task Performance}

The point of the behavioral task in this study was that object choice with a greater amount of reward during the choice cue or delay period could be made in a manner that was temporally dissociated from movement choice (Figure 1A; see section "Materials and Methods" for details). First, we confirmed that the monkeys could learn the association between a visual feature of the object (color or shape) and four levels of reward in every block of trials, and then choose the better target by releasing the button using the data recorded on neuronal activity (monkey 1, 119 days; monkey 2, 86 days) (Figure 2). The transition of the mean optimal choice ratio (the choice ratio of the target associated with the greater amount of reward) in the choice task across blocks indicated that the optimal choice ratio in both color-reward and shape-reward blocks increased after block change, whereas the optimal choice ratio corresponding to the previous block decreased in both monkeys (Figure 2A left). The reaction time (RT) from the onset of the first target to button release was faster when the monkey made an optimal choice with a larger reward than when it made an optimal choice with a smaller one, and the RTs for the larger reward became faster as the trials progressed following the block change (Figure 2A right). The monkeys could also make an optimal choice for any combination of first and second targets, predicting different amounts of reward (Figure 2B). In a quantitative analysis of the two monkeys, the choice probability of the first target and the mean RT for choosing the first target against the difference in reward amount between the first and second targets were calculated and showed consistent results with Figures 2A,B (Figures 2C,D). The choice probability of the first target against the difference exhibited a significant effect, and these choice behaviors did not differ significantly between the two monkeys (Figure 2C): two-way ANOVA, $F(5,2)=280.39, p<0.001$ for the difference in reward amount; $F(1,6)=0.94, p=0.38$ for monkey). RT was also significantly affected by the difference in reward amount (Figure 2D): one-way ANOVA, monkey 1, $F(5,9.99)$, $p<0.001$; monkey 2, $F(1,9.16), p<0.001$. In both monkeys, RT was significantly slower when the difference in reward amount was 1 than when it was 2 or 3 (Wilcoxon rank sum test with Bonferroni correction: monkey $1, p<0.001$ for reward 1 vs. reward 2, $p<0.001$ for reward 1 vs. reward 3, $p=0.0052$ for reward 2 vs. reward 3; monkey $2, p<0.001$ for reward 1 vs. reward 2, $p<0.001$ for reward 1 vs. reward 3, $p<0.001$ for reward 2 vs. reward 3 ). These behavioral results meant that the two monkeys learned the association between a visual feature of the object (color or shape) and four different amounts of reward and chose the target associated with the greater amount of reward by releasing the button according to the block change.

Next in this task, we aimed to specifically check whether monkeys made an object choice or not (Figures 2E,F). If monkeys could choose any one of two objects during choice cue or delay period in the choice task, the subsequent process would be the same as the instruction task, in which they simply chose the decided or instructed object by release the button during target presenting period. Subsequently, we compared the RT of the choice task and the instruction task (Figures 2E,F). RT in the instruction task was found to be faster than that in the choice task (Figure 2E, Wilcoxon 
A
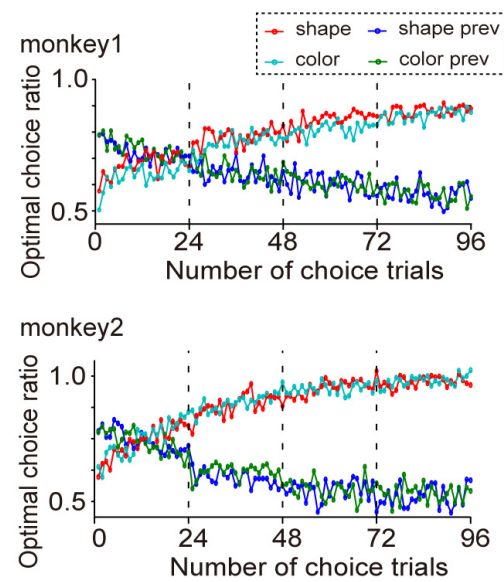
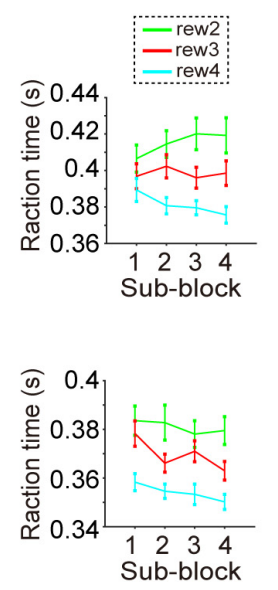

B

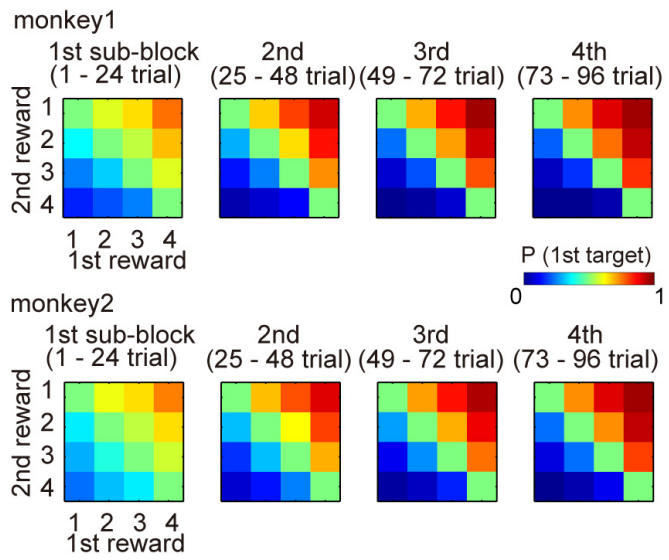

C monkey1

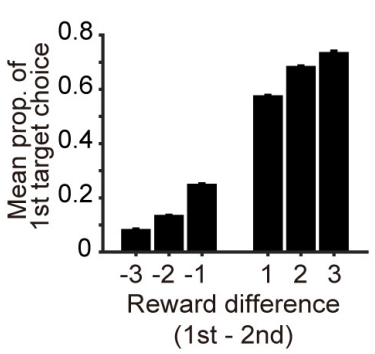

monkey2

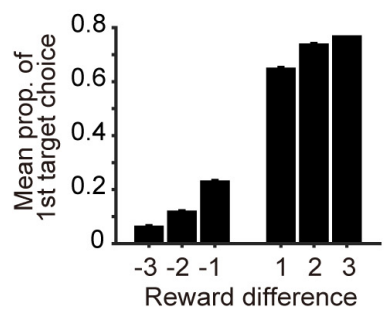

(1st - 2nd)
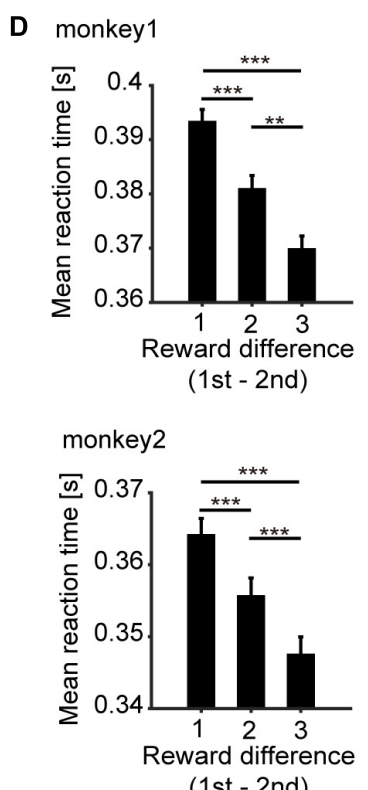

E

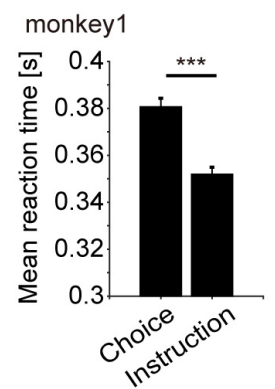

F monkey1
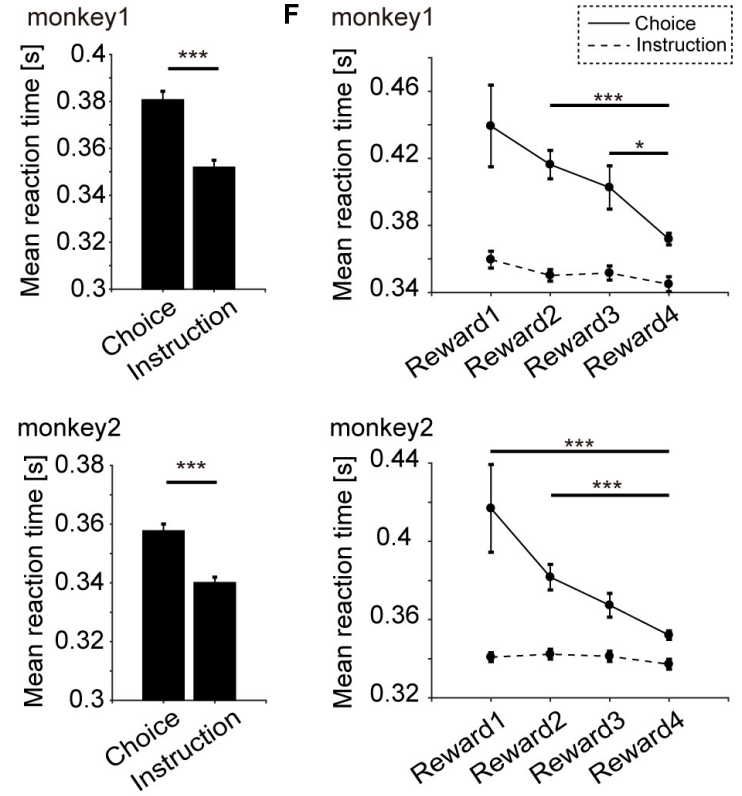

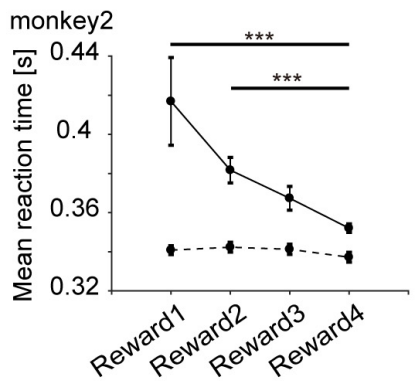

FIGURE 2 | Behavioral performance (monkeys 1 and 2: 119 days, 178 blocks; monkey 2: 86 days, 129 blocks). (A) Left: Transition of mean optimal choice ratio in the free-choice task after the block change with monkeys 1 and 2. Red and cyan indicate the optimal choice ratio in the current shape and color block, respectively. Blue and green indicate the optimal choice ratios in the previous shape and color blocks, respectively. Right: Mean reaction time (RT) and s.e.m. of the optimal choice trial vs. progress of subblocks (1st, 2nd, 3rd, and 4th) with monkeys 1 and 2. Cyan, red and green indicate RT when the chosen amount of reward was 4, 3 , and 2, respectively. (B) Mean probability of choosing the first target vs. the difference in the amount of reward with monkeys 1 and 2. (C) Mean probability of choosing the first target vs. the difference in reward amount (1st minus 2nd target) and s.e.m. for monkey 1 and monkey 2. (D) Mean RT and s.e.m. when the first target was chosen vs. the difference in the amount of reward (1st minus 2nd target) for monkeys 1 and 2. (E) Mean RT and s.e.m. of the choice task and the instruction task for monkey 1 and 2. (F) Mean RT and s.e.m. of the choice task and the instruction task for the four level of reward amount. Statistical test was performed by Wilcoxon rank sum test with Bonferroni correction. ${ }^{* * *} p<0.001,{ }^{* *} p<0.01,{ }^{*} p<0.05$.

rank sum test: monkey1, $p<0.001$; monkey2, $p<0.001)$. No significant effect was seen for RT in the instruction task for the four levels of reward, whereas RT in the choice task showed a significant effect (Figure 2F, one-way ANOVA, monkey1, $F(3,1.95), p=0.118$ for the instruction task, $F(3,11.4), p<0.001$ for the choice task; monkey2, $F(3,0.79)$, $p=0.502$ for the instruction task, $F(3,12.1), p<0.001$ for the choice task). These results showing different RT between the choice and the instruction tasks indicated that the behavioral analysis was unable to support the evidence that the monkeys made object choice.

\section{Population Neuronal Activity Evidence for Object Choice During the Choice Cue and Delay Periods}

Again, the salient feature of the behavioral task used in this study was that choice of object's visual feature, object choice, with 
greater amount of reward could be made during the choice cue or delay period, temporally dissociated from movement choice. However, we were unable to confirm this claim by behavioral analysis (Figures 2E,F). It is possible that the monkeys did not always need to use a strategy to choose one of two objects during the choice cue or delay period since they could choose both object and movement at the onset of the first target by remembering the two objects without making any object choice before. In the first step of our neuronal analysis, we aimed to examine whether the monkeys actually made an object choice during the choice cue or delay period by analyzing neuronal activities of all recorded neurons (monkey 1, $n=201$; monkey 2, $n=174$, Figure 3A). To this end, we searched the neuronal representation of "chosen object" during the choice cue or delay period by mutual information analysis and the bootstrap method. Because there was a possibility that mutual information of the chosen object had a spurious correlation with that of the choice cue (hereafter called the "offered objects"), we tried to identify whether the mutual information of the chosen object was significantly larger than the surrogate mutual information of the chosen object in which the information of the chosen object was randomized but the information of the offered object was kept (see section "Materials and Methods" for details). We calculated the summation of mutual information of chosen shape (or color) from all recorded neurons $(n=375)$ (Figure 3B) and performed the statistical test in each eight successive $0.2-\mathrm{s}$ windows from the onset of choice cue (threshold for significance: $p<0.05)$. We found significantly larger information of the chosen shape in the latter five windows and color in the second, third, and fourth windows than the surrogate information of chosen shape and color, respectively (Figure 3B and Table 1). We also checked whether there was mutual information of the offered object in distinction from surrogate mutual information of the offered object in which the information of the offered object was randomized but the information of the chosen object was kept. We found significantly larger information of the offered shape in the second to sixth windows and color in the third window than the surrogate information of offered shape and color, respectively (Figure 3C and Table 1). The significant representation of the chosen and offered object by population neuronal activities (summation of mutual information from all recorded neurons) suggested the presence of post- and pre-decision signals in the striatum along with evidence that the monkeys actually made an object choice during the choice cue or delay period prior to the movement choice.

Many previous studies reported that the striatum represents value signals. In the present task, the association between a visual feature of the object (shape or color) and four levels of reward was changed across blocks (Figure 1C), which might enable us to discriminate the "object-visual feature" from the "reward value" associated with the object. However, there was a possibility that the change of association across blocks was not enough to discriminate them, because we recorded neuronal activity across only two or three blocks (two or three times change of the association). Then, to check whether the information of the object-visual feature and the reward value could be regarded as different or not, we compared two surrogate distributions of the summation of mutual information for all recorded neurons $(n=375)$ (Figure 4). One was the surrogate distribution of the information of chosen (or offered) reward value, in which the information of the chosen (or offered) object was randomized but the information of the chosen (or offered) reward value was kept; the other was the distribution of information of the chosen (or offered) object, in which the information of the chosen (or offered) reward value was randomized but the information of the chosen (or offered) object was kept. Comparing the two distributions by ROC analysis for the classification of chosen object and chosen reward value in 0.2-s windows showing the significance in Figure 3B, the AUC for chosen shape was 0.74, $0.76,0.69,0.66$, and 0.61 (Figure 4A upper, $p<0.001$ for all five windows, Mann-Whitney $U$-test for difference between the distribution of AUCs and 0.5; see section "Materials and Methods" for statistics) and AUC for chosen color was 0.69, 0.85, and 0.78 (Figure 4A lower, $p<0.001$ for all three windows, Mann-Whitney $U$-test). For the classification of offered object and reward value, the AUC for offered shape was 0.81, 0.71, 0.59 , and 0.66 (Figure 4B upper, $p<0.001$ for all four windows, Mann-Whitney $U$-test), and for offered color it was 0.50 (Figure 4B lower, $p=0.115$, Mann-Whitney $U$-test). These results indicated that the information of chosen shape, chosen color and offered shape were larger than that of value, whereas offered color and value were not discriminated well in the present task and data.

\section{Neuronal Representation in Relation to Object Choice at the Single-Neuron Level}

As population neuronal activities indicating that the monkeys actually made object choices were confirmed (Figures 3B,C), we investigated the neuronal representations of offered object and chosen object during the choice cue or delay period at the singleneuron level. Perievent time histogram (PETH) of an example of averaged activity of a single neuron aligned at the onset of choice cue in a choice task (Figure 5A left) revealed differential activity according to the shape combination of the offered object in the 0-0.4-s window from the onset of choice cue (one-way ANOVA, $p=0.0073$ ). The mutual information of offered shape of this neuron in the same window was significantly larger than that of surrogate information, in which the information of offered shape was randomized and the information of chosen shape was kept (bootstrap method, $p=0.02$; see section "Materials and Methods" for statistics). Here, we defined this type of neuron as an "offered shape-type neuron." This offered shape-type neuron did not show differential activity according to the offered shape in the instruction task ( $p=0.35$ ) (Figure 5A right). To confirm the distribution of offered object (shape or color)-type neurons in choice task, instruction task, or both tasks, we calculated the proportion of offered object-type neurons for the choice task and the instruction task separately. For both offered shapeand color-type neurons, the proportion for both tasks exceeded the chance level that was expected from the proportion for choice and instruction task each $\left[x^{2}(1)=11.945, p<0.001\right.$ for offered color, $x^{2}(1)=6.316, p=0.012$ for offered shape, 

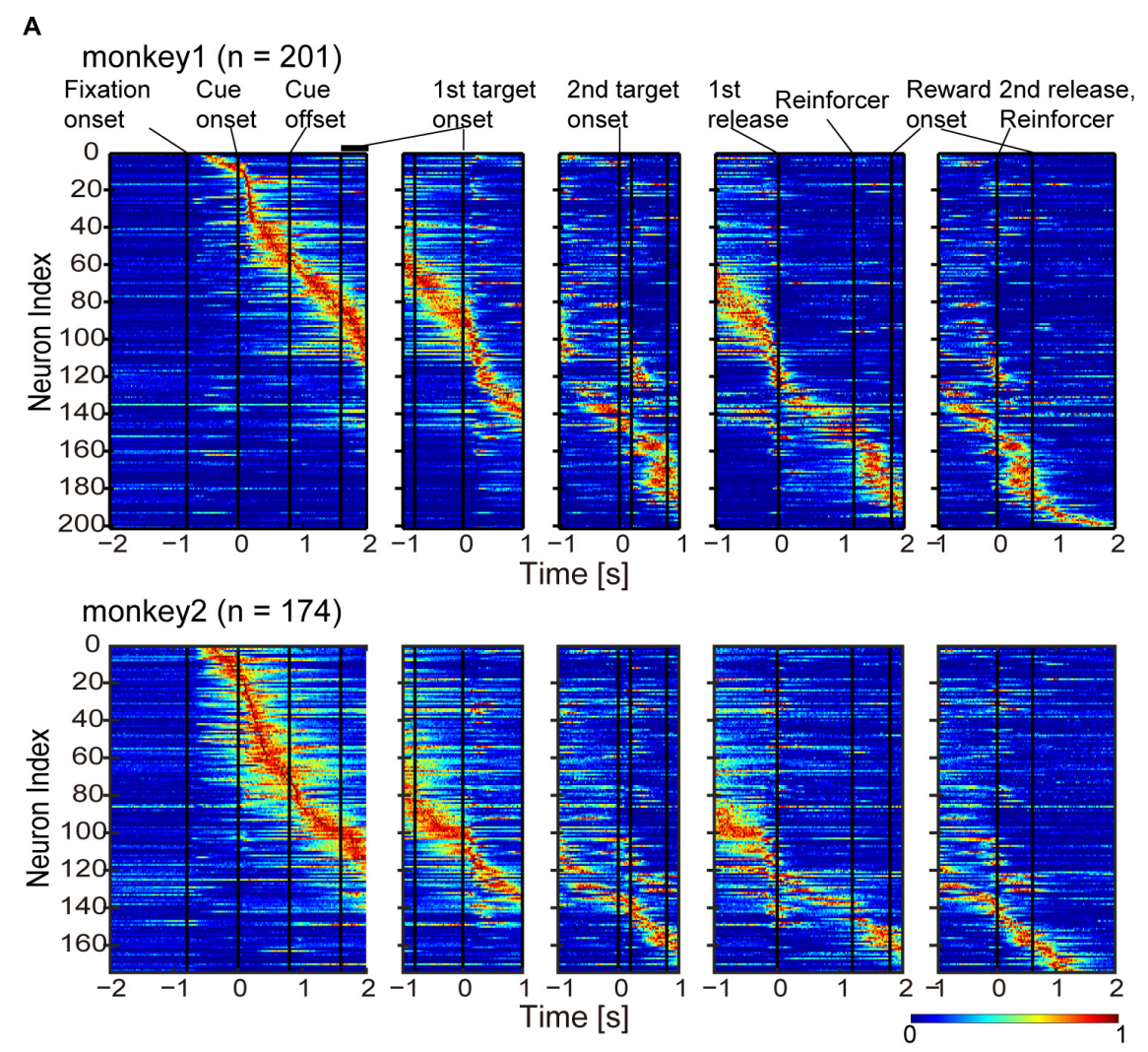

B
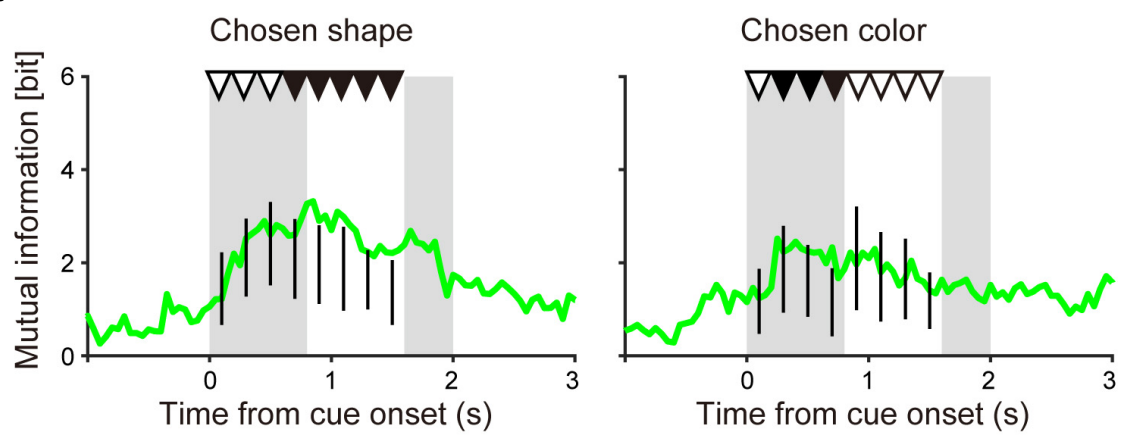

C
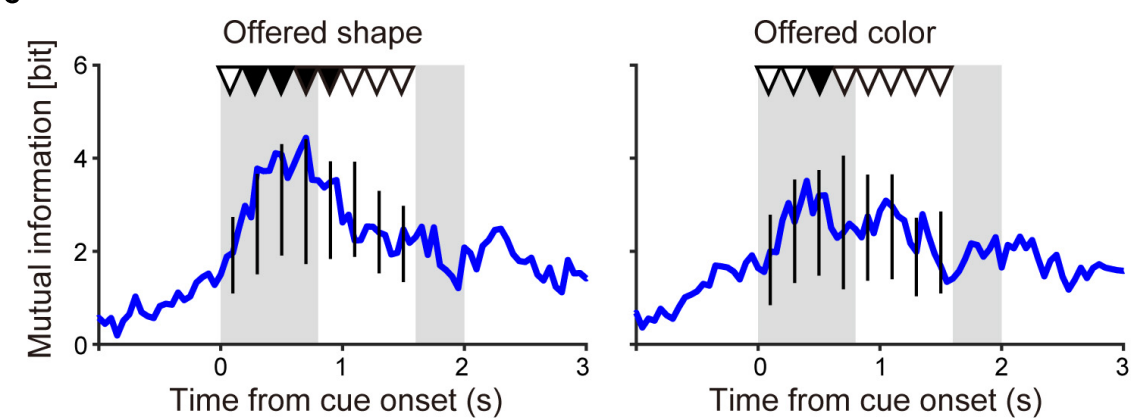

FIGURE 3 | Population neuronal activity evidence for object choice during the choice cue and delay periods. (A) Normalized firing rate of monkeys 1 and 2 . The firing rate was normalized for each neuron with the maximum firing rate in a 0.05-s window. (B) Time course of mutual information of chosen object of shape (left) and color (right) for all recorded neurons $(n=375)$ aligned at onset of choice cue. Vertical black lines indicate the surrogate distribution of the mutual information of chosen shape $\left(n_{\text {shuffles }}=10,000\right.$ ) in successive eight 0.2-s windows from onset of choice cue. First and second gray shadows indicate the cue presentation period ( $0.4 \mathrm{~s})$ and onset of the first target following variable cue delay period, respectively. Upper black and white triangles indicate significant differences between real mutual information and surrogate information and non-significant differences between them, respectively. (C) Same as (B) but for the information of offered object. 
TABLE $1 \mid P$-values for the information of chosen and offered objects related to Figure 3.

\begin{tabular}{|c|c|c|c|c|c|c|c|c|c|}
\hline \multirow[t]{2}{*}{ Object } & \multirow[t]{2}{*}{ Type } & \multicolumn{8}{|c|}{ Time from onset of choice cue (s) } \\
\hline & & $0-0.2$ & $0.2-0.4$ & $0.4-0.6$ & $0.6-0.8$ & $0.8-1.0$ & $1.0-1.2$ & $1.2-1.4$ & $1.4-1.6$ \\
\hline \multirow[t]{2}{*}{ Chosen } & Shape & 0.486 & 0.058 & 0.090 & 0.006 & 0 & 0 & 0.002 & 0 \\
\hline & Color & 0.282 & 0.039 & 0.0013 & 0 & 0.418 & 0.244 & 0.382 & 0.147 \\
\hline \multirow[t]{2}{*}{ Offered } & Shape & 0.256 & 0 & 0.003 & 0 & 0.036 & 0.965 & 0.332 & 0.128 \\
\hline & Color & 0.100 & 0.233 & 0.018 & 0.351 & 0.168 & 0.054 & 0.260 & 0.853 \\
\hline
\end{tabular}

Chi-squared test, Figure 5B]. An example of another neuron (Figure 5C left) showed differential activity according to the shape of the chosen object in the 1.2-1.6-s window from onset of choice cue in the choice task (one-way ANOVA, $p=0.0068$ ). The mutual information of chosen shape of this neuron in the same window was significantly larger than the surrogate information, in which the information of chosen shape was randomized and the information of offered shape was kept $(p=0.01)$. We defined this type of neuron as a "chosen shapetype neuron." This chosen shape-type neuron did not show differential activity according to chosen shape in the instruction task $(p=0.56)$ (Figure 5C right). Similar to the offered objecttype neurons, we calculated the proportion of chosen objecttype neurons for the choice and instruction tasks separately. For chosen shape-type neurons, the proportion for both tasks exceeded the chance level that was expected from the proportion for only choice and instruction tasks, whereas the proportion for chosen color-type neurons did not $\left[x^{2}(1)=10.133, p=0.015\right.$ for chosen shape, $x^{2}(1)=2.293, p=0.13$ for chosen color, Chisquared test, Figure 5D]. These results indicated the presence of offered and chosen object signals at the single-neuron level during the choice cue or delay period, and these signals were represented by all three types of neurons (choice, instruction, and both tasks).

In the present task, following object choice the monkey needed to release the button during the first or second target-presenting period (Figure 1). PETH of an example of averaged activity of a single neuron aligned at onset of choice cue (Figure 6A) revealed differential activity according to first or second release in the 0.8-s window after onset of the first target (one-way ANOVA, $\left.p=2.2 \times 10^{-36}\right)$. We defined this type of neuron as a "movement-type neuron." Mutual information analysis using task condition of movement ( 1 st and 2 nd release) revealed that the information was not evident during the choice cue and delay period, but it was strongly represented after onset of the first target (Figure 6B). We checked the overlap between offered object-type or chosen object-type neurons with movement-type neurons (Figures 6C,D). For both offered shape- and colortype neurons, the proportion overlapping with movement-type neurons was around or below the chance level $\left[x^{2}(1)=19.189\right.$, $p<0.001$ for offered shape, $x^{2}(1)=0.354, p=0.552$ for offered color, Chi-squared test, Figure 6C]. For chosen shape- or colortype neurons, similar to the offered type neurons, the proportion of overlap with movement-type neurons was around or below the chance level $\left[x^{2}(1)=1.132, p=0.843\right.$ for chosen shape, $x^{2}(1)=4.772, p=0.0289$ for chosen color, Chi-squared test,
Figure 6D]. These results indicated that the object and movement signals were represented by separate neurons.

\section{DISCUSSION}

To study neuronal representation in relation to object choice, which does not include physical action, in the striatum, we designed a behavioral task, in which object choice could be temporally dissociated from movement choice, and trained two monkeys in the task (Figures 1, 2). We recorded 375 striatal PANs of the two monkeys (Figure 3A). We calculated the mutual information using the task condition of the chosen object for all recorded neurons and performed statistical tests using the bootstrap method, and found that population striatal activities represented the information of the chosen object in distinction from the offered object during the choice cue and delay period, which indicated that the monkeys actually made an object choice during the task (Figure 3B and Table 1). We also found the neuronal representation of offered object in distinction from chosen object during the period (Figure 3C and Table 1). For the activity of individual neurons, we investigated the neuronal representations of the offered object and chosen object and identified offered object- and chosen object-type neurons (Figure 5). Furthermore, we also identified that the movementtype neurons discriminated between the first and second release during the first target-presenting period (Figures 6A,B). Most offered object- or chosen object-type neurons did not overlap with movement-type neurons (Figures 6C,D). These findings suggested that the presence of object choice-related signals in the striatum and their signals were represented by other neurons related to movement.

Previous studies investigated the involvement of the striatum in action choice using behavioral tasks, in which the alternatives for choice included both motor and non-motor factors simultaneously, e.g., alternatives predicting reward values and motor direction (Takikawa et al., 2002; Samejima et al., 2005; Lau and Glimcher, 2008; Tai et al., 2012). Although some studies have examined the neuronal activity of the striatum in relation to reward expectation without the motor aspect (Lauwereyns et al., 2002; Cromwell and Schultz, 2003), these behavioral tasks did not include choices of alternatives. A unique feature of this study is that object choice (choice for visual feature) could be made during the choice cue or the delay periods, which was temporally dissociated from movement choice. Furthermore, in this task, because two objects were presented in $2 \times 2$ form spatially in 
A Chosen shape vs. value

shape | shuffling value
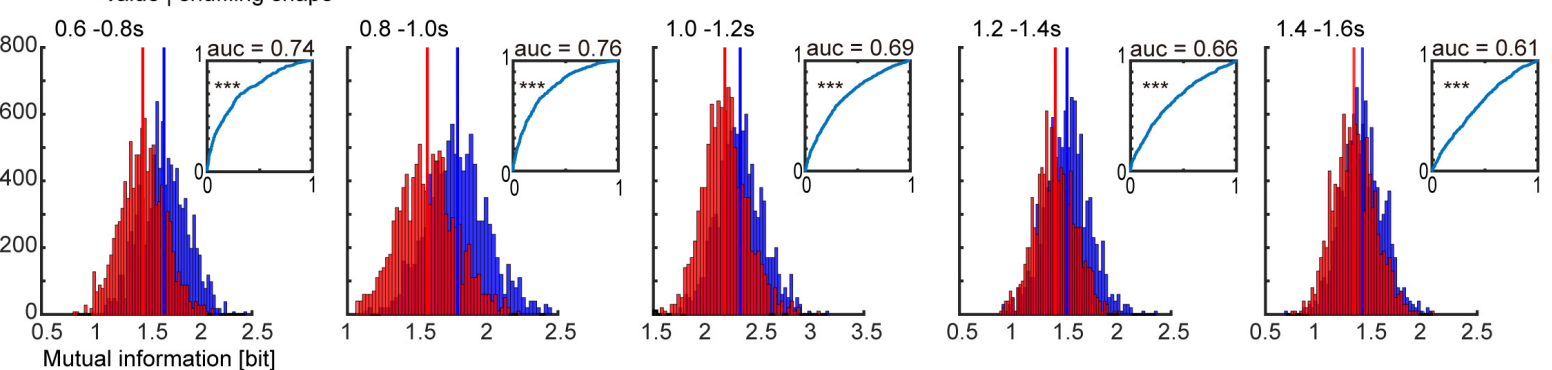

Chosen color vs. value

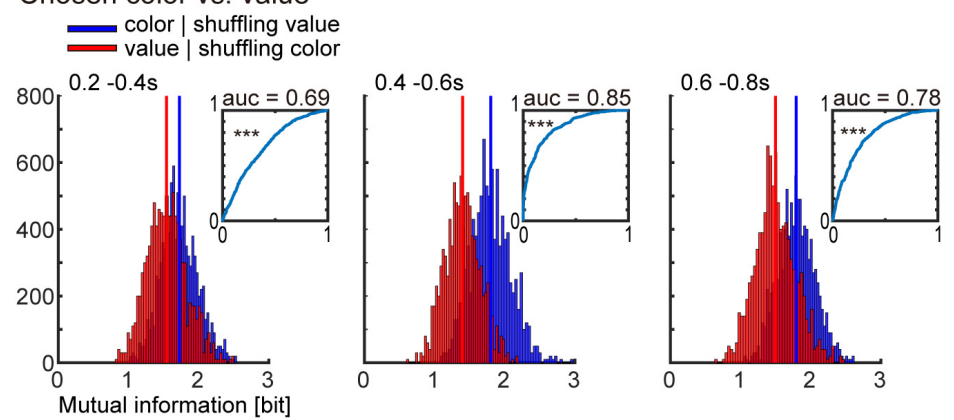

B
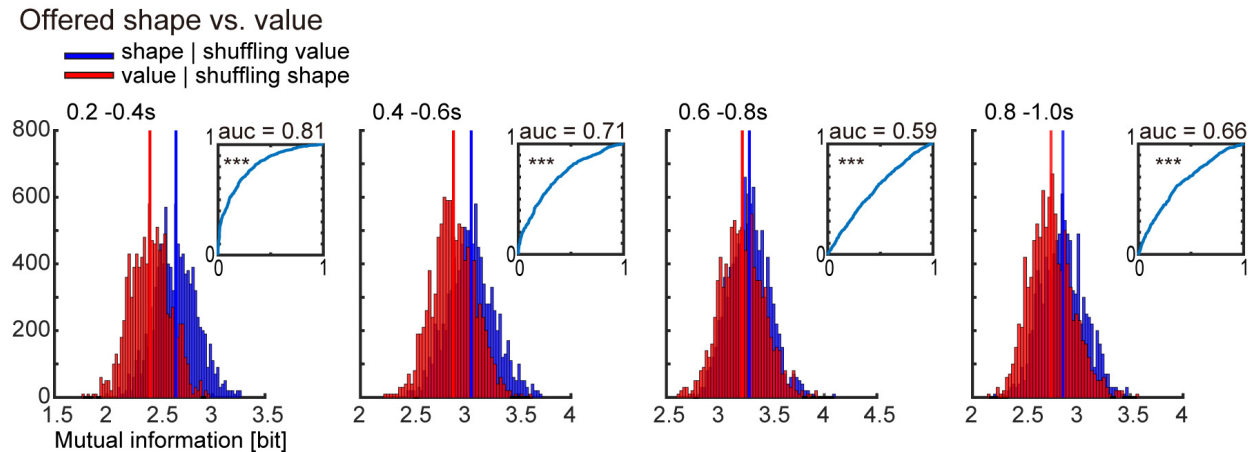

Offered color vs. value

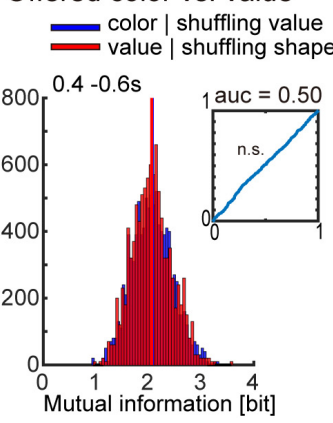

FIGURE 4 | Receiver operating characteristic (ROC) analysis for classification of object-visual feature and object-reward value. (A) Surrogate distribution of chosen object-visual feature and object-reward value. Upper: Surrogate distribution of chosen shape (blue) and value (red) generated from shuffling of chosen value and shape, respectively (10,000 shuffles) in 0.2-s window showing significance in Figure 3B left. Inset indicates the ROC curve for classification of chosen shape. Horizontal and vertical lines indicate the false positive and true positive rates, respectively. AUC is the area under the curve. The statistical test for the AUC was performed by Mann-Whitney U-test (see section "Materials and Methods" for details). Lower: Surrogate distribution of chosen color (blue) and value (red) generated from shuffled chosen value and shape, respectively (10,000 shuffles) in 0.2-s window showing significance in Figure 3B right. (B) Same as (A) but for offered object (shape and color) and value. ${ }^{* * *} p<0.001$. 


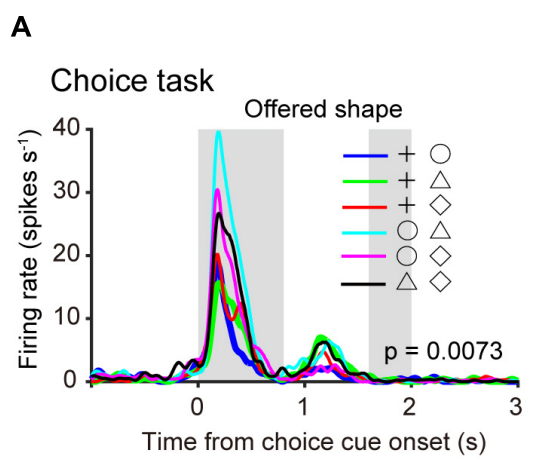

C

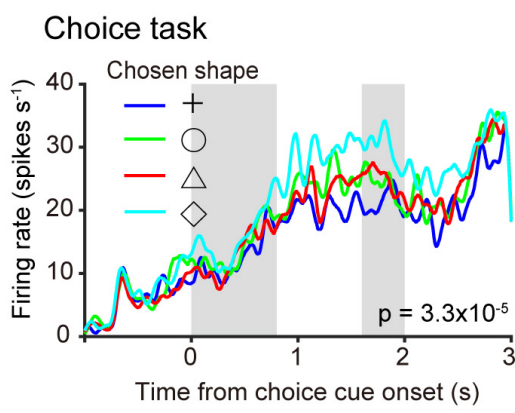

Instruction task

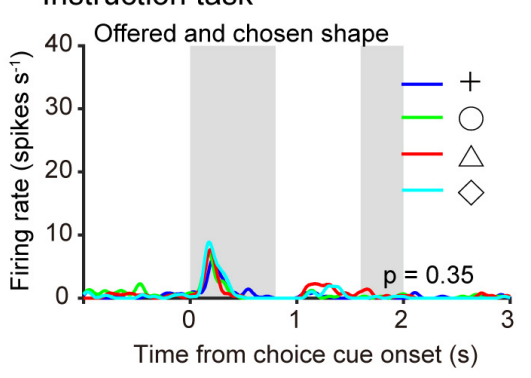

B

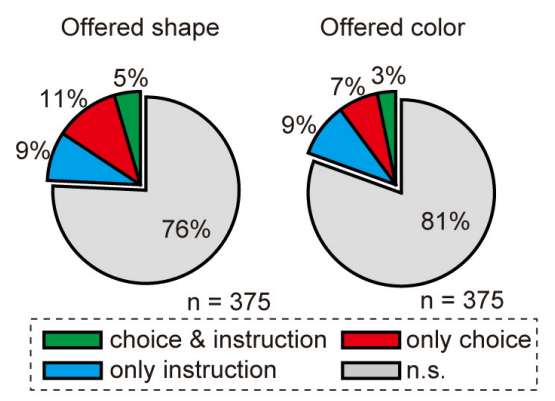

D

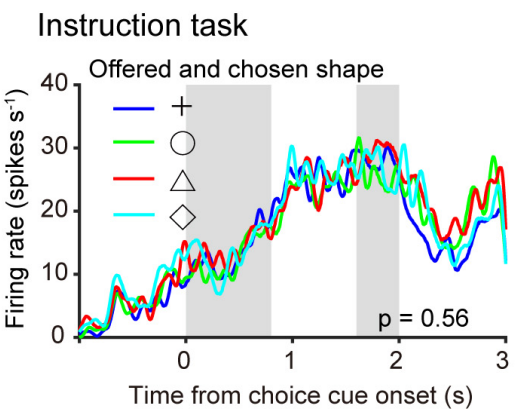

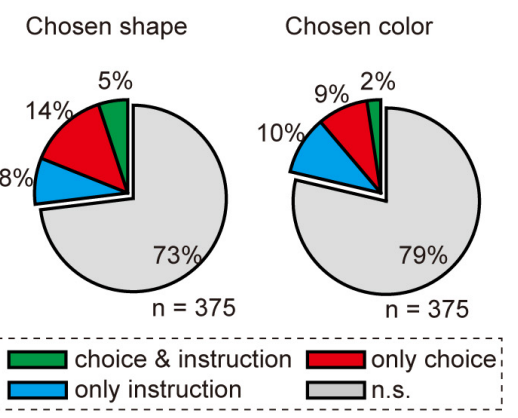

FIGURE 5 | Neuronal representation in relation to object choice at the single-neuron level. (A) Left: Example of time course of averaged single neuronal activity representing the offered shape, defined as an offered shape-type neuron (0-0.4-s window from onset of choice cue; one-way ANOVA, $p=0.0073$; bootstrap, $p=0.02)$. First and second gray shadows indicate cue presentation period ( $0.4 \mathrm{~s})$ and onset of the first target following a variable cue delay period (0.8-1.2 s), respectively. Right: Firing rate, in the same neuron, sorted by offered and chosen shape in instruction task (one-way ANOVA, $p=0.35$ ). (B) Left and right pie charts show the proportion of offered shape- and color-type neurons among all recorded neurons $(n=375)$. Green, orange, and blue indicate significant differences $(p<0.05)$ in both choice and instruction task, choice task only, and instruction task only, respectively. (C) Left: Example of average time course of single neuronal activity representing the chosen shape, defined as a chosen shape-type neuron (1.2-1.6-s window from onset of choice cue; one-way ANOVA, $p=3.3 \times 10^{-5}$; bootstrap, $p=0.01$ ). First and second gray shadows indicate cue presentation period $(0.4 \mathrm{~s})$ and onset of the first target following a variable cue delay period (0.8-1.2 s), respectively. Right: Firing rate, in the same neuron, sorted by offered and chosen shape in instruction task (one-way ANOVA, $p=0.56)$. (D) Same as (A) but for chosen object type neurons.

four corners, spatial information of the two objects was hashed, which means that the object choice could not be made for spatialspecific position. This is the first study to reveal the neuronal representations in the striatum in relation to object choice by designing and adopting a behavioral task, in which the period used to make an object choice is explicitly extracted.

We were unable to confirm the evidence that monkeys actually made object choice through behavioral analysis (Figures 2E,F). However, in neuronal analysis, we found that the neuronal representation of chosen object was distinct from offered object during choice cue and delay period (Figure 3B), which indicated that object choice was made. We also found the neuronal representation of offered object during the period (Figure 3C). These representations of chosen and offered object were regarded as post- and pre-decision signals without physical action, respectively. In fact, chosen shape and offered shape in Figure 3 showed a dynamically significant representation in the order of the decision process (from pre- to post-decision signals). For the color representation, we were unable to explain the temporal dynamics like shape information. Further research is required to reveal the mechanisms of different signals such as shape, color, and offered and chosen information were temporally represented and related each other.

In the present study, we found the neuronal representations of the object-visual feature (chosen shape, color, and offered shape) rather than that of the reward value (Figure 4). Although this seems like a paradoxical result in comparison with previous studies reporting value-related signals in the striatum, some previous studies (Samejima et al., 2005; Lau and Glimcher, 2008) have reported that there are lots of non-value neurons that show a differential response according to movement direction when animals make a decision, as well as value type neurons. Although there is a discrepancy between present and previous tasks regarding whether the alternatives for choice include physical action or not, the non-value neurons in previous and present studies could be interpreted as the same type of neurons that represent the option signal without value. Therefore, the results of neuronal representation for object-visual feature in this study are consistent with those of previous studies.

Anatomically, the striatum has inputs from various cerebral cortical areas, including the prefrontal, higher-order motor, and primary motor cortex, and it returns these inputs via the thalamus 


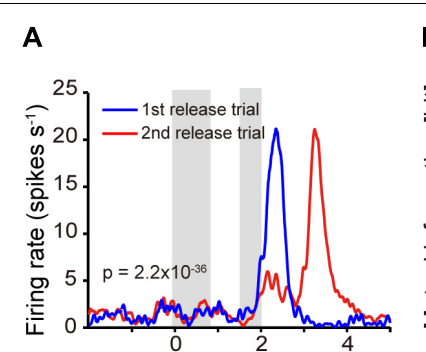

Time from choice cue onset (s)

C
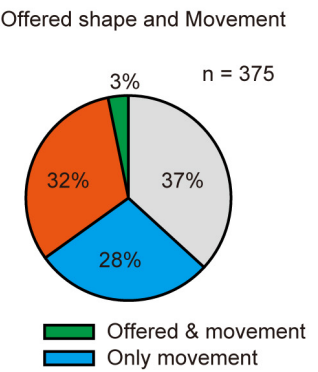

D
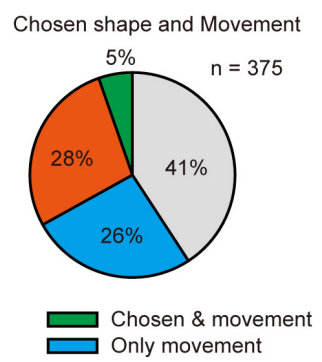

\section{B}

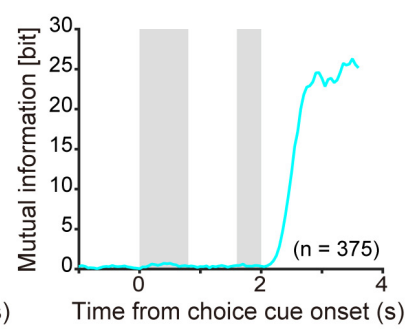

Offered color and Movement

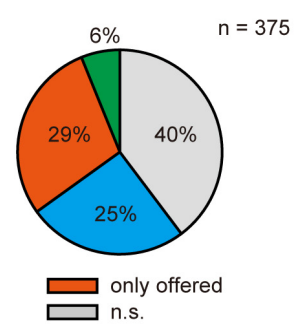

Chosen color and Movement

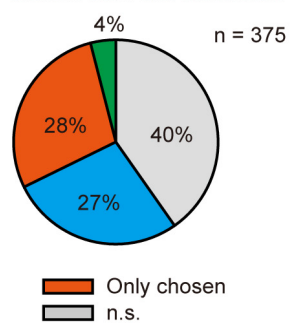

FIGURE 6 | Neuronal representation in relation to movement choice. (A) Example of time course of averaged single neuronal activity representing movement choice (0-0.8-s window from onset of the first target; one-way ANOVA, $\left.p=2.2 \times 10^{-36}\right)$. First and second gray shadows indicate cue presentation period $(0.4 \mathrm{~s})$ and onset of the first target following a variable cue delay period (0.8-1.2 s), respectively. (B) Time course of mutual information of movement for all recorded neurons $(n=375)$ aligned at onset of choice cue. (C) Overlap offered shape- (left) and color- (right) type neurons with movement-type neurons among all recorded neurons $(n=375)$. Green, orange, and blue indicate significant differences $(p<0.05)$ in both offered shape (or color) and movement, offered shape (or color) only, and movement only, respectively. (D) Same as (C) but for chosen object type neurons. color or shape is represented in the prefrontal cortex (Divac et al., 1967; Levy et al., 1997; Sakagami and Tsutsui, 1999; Lauwereyns et al., 2001). The anterior caudate receives input mainly from the prefrontal cortex, including the dorsolateral prefrontal cortex, the OFC, the anterior cingulate cortex, and the SEF (Yeterian and Van Hoesen, 1978; Selemon and GoldmanRakic, 1985; Alexander et al., 1986; Haber, 2016). Considering the anatomical connections from the prefrontal cortex to the anterior caudate and its neuronal representation, including the present results, object choice could be made through the prefrontal loop including the anterior caudate and the prefrontal cortex by using information about its non-spatial value and attributes of object. However, human functional magnetic resonance imaging (fMRI) studies have reported the presence of object choice signals in the ventromedial prefrontal cortex (Wunderlich et al., 2010; Hare et al., 2011), projecting mainly to the ventral striatum. The neuronal representations for the object choice in each striatal subarea need to be investigated. On the other hand, for movement choice, several studies have suggested that the premotor cortex plays an important role (Schieber, 2000; Lauwereyns et al., 2002; Nakayama et al., 2008; Thura and Cisek, 2014). The present study revealed the presence of movement-related signals (Figure 6). Considering the anatomical connections, their movement-related signals might be processed within the premotor loop. It will be necessary to classify the distribution of neuronal representation in relation to object choice and movement choice based on the striatal subregion.

Taken together, the investigation of object choice has so far concentrated on the cortex. Our results reveal the neuronal representation in relation to object choice in the striatum and show the importance of cortico-basal ganglia circuits in decision-making.

\section{DATA AVAILABILITY STATEMENT}

All data that support the findings of this study are available from the Lead Contact (satoshi.nonomura@gmail.com) upon reasonable request.

\section{ETHICS STATEMENT}

All experiments were approved by the Animal Research Ethics Committee of Tamagawa University (animal experiment protocol H21/27-14) and were carried out in accordance with the Fundamental Guidelines for Proper Conduct of Animal Experiments and Related Activities in Academic Research Institutions [Ministry of Education, Culture, Sports, Science and Technology (MEXT) of Japan] and the Guidelines for Animal Experimentation in Neuroscience (Japan Neuroscience Society). All surgical procedures were performed under appropriate anesthesia, and all efforts were made to minimize suffering. Our procedures for primate animal experiments were established in previous studies at Tamagawa University (Nakayama et al., 2008; Yamagata et al., 2009; Hashimoto et al., 2010; Saga et al., 2011; Arimura et al., 2013). 


\section{AUTHOR CONTRIBUTIONS}

SN and KS designed and performed the research, analyzed the data, and wrote the manuscript.

\section{FUNDING}

This work was supported by JSPS KAKENHI Grant Number JP12J56654 and MEXT KAKENHI Grant Numbers

\section{REFERENCES}

Alexander, G. E., DeLong, M. R., and Strick, P. L. (1986). Parallel organization of functionally segregated circuits linking basal ganglia and cortex. Annu. Rev. Neurosci. 9, 357-381. doi: 10.1146/annurev.ne.09.030186.002041

Aosaki, T., Tsubokawa, H., Ishida, A., Watanabe, K., Graybiel, A. M., and Kimura, M. (1994). Responses of tonically active neurons in the primate's striatum undergo systematic changes during behavioral sensorimotor conditioning. J. Neurosci. 14, 3969-3984. doi: 10.1523/JNEUROSCI.14-0603969.1994

Arimura, N., Nakayama, Y., Yamagata, T., Tanji, J., and Hoshi, E. (2013) Involvement of the globus pallidus in behavioral goal determination and action specification. J. Neurosci. 33, 13639-13653. doi: 10.1523/JNEUROSCI.1620-13. 2013

Beste, C., Saft, C., Andrich, J., Gold, R., and Falkenstein, M. (2008). Stimulusresponse compatibility in Huntington's disease: a cognitive-neurophysiological analysis. J. Neurophysiol. 99, 1213-1223. doi: 10.1152/jn.01152.2007

Cai, X., Kim, S., and Lee, D. (2011). Heterogeneous coding of temporally discounted values in the dorsal and ventral striatum during intertemporal choice. Neuron 69, 170-182. doi: 10.1016/j.neuron.2010.11.041

Cai, X., and Padoa-Schioppa, C. (2014). Contributions of orbitofrontal and lateral prefrontal cortices to economic choice and the good-to-action transformation. Neuron 81, 1140-1151. doi: 10.1016/j.neuron.2014.01.008

Chen, X., and Stuphorn, V. (2015). Sequential selection of economic good and action in medial frontal cortex of macaques during value-based decisions. eLife 4:e09418. doi: 10.7554/eLife.09418

Cisek, P. (2012). Making decisions through a distributed consensus. Curr. Opin. Neurobiol. 22, 927-936. doi: 10.1016/j.conb.2012.05.007

Cromwell, H. C., and Schultz, W. (2003). Effects of expectations for different reward magnitudes on neuronal activity in primate striatum. J. Neurophysiol. 89, 2823-2838. doi: 10.1152/jn.01014.2002

Divac, I., Rosvold, H. E., and Szwarcbart, M. K. (1967). Behavioral effects of selective ablation of the caudate nucleus. J. Comp. Physiol. Psychol. 63, 184-190. doi: $10.1037 / \mathrm{h} 0024348$

Flaherty, A. W., and Graybiel, A. M. (1993). Two input systems for body representations in the primate striatal matrix: experimental evidence in the squirrel monkey. J. Neurosci. 13, 1120-1137. doi: 10.1523/jneurosci.13-0301120.1993

Frank, M. J., Seeberger, L. C., and O’Reilly, R. C. (2004). By carrot or by stick: cognitive reinforcement learning in parkinsonism. Science 306, 1940-1943. doi: $10.1126 /$ science. 1102941

Grabenhorst, F., Hernadi, I., and Schultz, W. (2012). Prediction of economic choice by primate amygdala neurons. Proc. Natl. Acad. Sci. U.S.A. 109, 18950-18955. doi: $10.1073 /$ pnas. 1212706109

Graybiel, A. M., and Rauch, S. L. (2000). Toward a neurobiology of obsessivecompulsive disorder. Neuron 28, 343-347. doi: 10.1016/s0896-6273(00) 00113-6

Haber, S. N. (2016). Corticostriatal circuitry. Dialogues Clin. Neurosci. 18, 7-21.

Haber, S. N., and Knutson, B. (2010). The reward circuit: linking primate anatomy and human imaging. Neuropsychopharmacology 35, 4-26. doi: 10.1038/npp. 2009.129

Hare, T. A., Schultz, W., Camerer, C. F., O’Doherty, J. P., and Rangel, A. (2011). Transformation of stimulus value signals into motor commands during simple
JP20680020, JP22120514, JP24120716, JP16H01725, JP19K16300, and JP19H04988.

\section{ACKNOWLEDGMENTS}

We thank E. Hoshi, J. Tanji, K. Doya, and members of the Samejima and Hoshi laboratories for general discussion and technical support; Y. Sakai and K. Mitani for help with statistical analysis; and K. Hamatani for administrative support.

choice. Proc. Natl. Acad. Sci. U.S.A. 108, 18120-18125. doi: 10.1073/pnas. 1109322108

Hashimoto, M., Takahara, D., Hirata, Y., Inoue, K., Miyachi, S., Nambu, A., et al. (2010). Motor and non-motor projections from the cerebellum to rostrocaudally distinct sectors of the dorsal premotor cortex in macaques. Eur. J. Neurosci. 31, 1402-1413. doi: 10.1111/j.1460-9568.2010.07151.x

Hikosaka, O., Sakamoto, M., and Usui, S. (1989). Functional properties of monkey caudate neurons. I. activities related to saccadic eye movements. J. Neurophysiol. 61, 780-798. doi: 10.1152/jn.1989.61.4.780

Lau, B., and Glimcher, P. W. (2008). Value representations in the primate striatum during matching behavior. Neuron 58, 451-463. doi: 10.1016/j.neuron.2008.02.021

Lauwereyns, J., Sakagami, M., Tsutsui, K., Kobayashi, S., Koizumi, M., and Hikosaka, O. (2001). Responses to task-irrelevant visual features by primate prefrontal neurons. J. Neurophysiol. 86, 2001-2010. doi: 10.1152/jn.2001.86.4. 2001

Lauwereyns, J., Takikawa, Y., Kawagoe, R., Kobayashi, S., Koizumi, M., Coe, B., et al. (2002). Feature-based anticipation of cues that predict reward in monkey caudate nucleus. Neuron 33, 463-473. doi: 10.1016/s0896-6273(02)00571-8

Levy, R., Friedman, H. R., Davachi, L., and Goldman-Rakic, P. S. (1997). Differential activation of the caudate nucleus in primates performing spatial and nonspatial working memory tasks. J. Neurosci. 17, 3870-3882. doi: 10.1523/ jneurosci.17-10-03870.1997

Mink, J. W. (2003). The basal ganglia and involuntary movements: impaired inhibition of competing motor patterns. Arch. Neurol. 60, 1365-1368. doi: 10.1001/archneur.60.10.1365

Nakayama, Y., Yamagata, T., Tanji, J., and Hoshi, E. (2008). Transformation of a virtual action plan into a motor plan in the premotor cortex. J. Neurosci. 28, 10287-10297. doi: 10.1523/JNEUROSCI.2372-08.2008

Nonomura, S., Nishizawa, K., Sakai, Y., Kawaguchi, Y., Kato, S., Uchigashima, M., et al. (2018). Monitoring and updating of action selection for goal-directed behavior through the striatal direct and indirect pathways. Neuron 99, 1302. e5-1314.e5. doi: 10.1016/j.neuron.2018.08.002

Optican, L. M., and Richmond, B. J. (1987). Temporal encoding of twodimensional patterns by single units in primate inferior temporal cortex. III. Information theoretic analysis. J. Neurophysiol. 57, 162-178. doi: 10.1152/jn. 1987.57.1.162

Padoa-Schioppa, C. (2009). Range-adapting representation of economic value in the orbitofrontal cortex. J. Neurosci. 29, 14004-14014. doi: 10.1523/ JNEUROSCI.3751-09.2009

Padoa-Schioppa, C. (2011). Neurobiology of economic choice: a good-based model. Annu. Rev. Neurosci. 34, 333-359. doi: 10.1146/annurev-neuro-061010113648

Padoa-Schioppa, C., and Assad, J. A. (2006). Neurons in the orbitofrontal cortex encode economic value. Nature 441, 223-226. doi: 10.1038/nature 04676

Saga, Y., Hirata, Y., Takahara, D., Inoue, K., Miyachi, S., Nambu, A., et al. (2011). Origins of multisynaptic projections from the basal ganglia to rostrocaudally distinct sectors of the dorsal premotor area in macaques. Eur. J. Neurosci. 33, 285-297. doi: 10.1111/j.1460-9568.2010.07492.x

Sakagami, M., and Tsutsui, K. (1999). The hierarchical organization of decision making in the primate prefrontal cortex. Neurosci. Res. 34, 79-89. doi: 10.1016/ s0168-0102(99)00038-3 
Samejima, K., and Doya, K. (2007). Multiple representations of belief states and action values in corticobasal ganglia loops. Ann. N. Y. Acad. Sci. 1104, 213-228. doi: 10.1196/annals.1390.024

Samejima, K., Ueda, Y., Doya, K., and Kimura, M. (2005). Representation of actionspecific reward values in the striatum. Science 310, 1337-1340. doi: 10.1126/ science. 1115270

Schieber, M. H. (2000). Inactivation of the ventral premotor cortex biases the laterality of motoric choices. Exp. Brain Res. 130, 497-507. doi: 10.1007/ s002219900270

Selemon, L. D., and Goldman-Rakic, P. S. (1985). Longitudinal topography and interdigitation of corticostriatal projections in the rhesus monkey. J. Neurosci. 5, 776-794. doi: 10.1523/jneurosci.05-03-00776.1985

So, N. Y., and Stuphorn, V. (2010). Supplementary eye field encodes option and action value for saccades with variable reward. J. Neurophysiol. 104, 2634-2653. doi: 10.1152/jn.00430.2010

Tai, L. H., Lee, A. M., Benavidez, N., Bonci, A., and Wilbrecht, L. (2012). Transient stimulation of distinct subpopulations of striatal neurons mimics changes in action value. Nat. Neurosci. 15, 1281-1289. doi: 10.1038/nn.3188

Takikawa, Y., Kawagoe, R., Itoh, H., Nakahara, H., and Hikosaka, O. (2002). Modulation of saccadic eye movements by predicted reward outcome. Exp. Brain Res. 142, 284-291. doi: 10.1007/s00221-001-0928-1

Thura, D., and Cisek, P. (2014). Deliberation and commitment in the premotor and primary motor cortex during dynamic decision making. Neuron 81, 1401-1416. doi: 10.1016/j.neuron.2014.01.031
Wunderlich, K., Rangel, A., and O’Doherty, J. P. (2010). Economic choices can be made using only stimulus values. Proc. Natl. Acad. Sci. U.S.A. 107, 15005-15010. doi: $10.1073 /$ pnas. 1002258107

Yamada, H., Matsumoto, N., and Kimura, M. (2007). History- and current instruction-based coding of forthcoming behavioral outcomes in the striatum. J. Neurophysiol. 98, 3557-3567. doi: 10.1152/jn.00779.2007

Yamagata, T., Nakayama, Y., Tanji, J., and Hoshi, E. (2009). Processing of visual signals for direct specification of motor targets and for conceptual representation of action targets in the dorsal and ventral premotor cortex. J. Neurophysiol. 102, 3280-3294. doi: 10.1152/jn.00452.2009

Yeterian, E. H., and Van Hoesen, G. W. (1978). Cortico-striate projections in the rhesus monkey: the organization of certain cortico-caudate connections. Brain Res. 139, 43-63. doi: 10.1016/0006-8993(78)90059-8

Conflict of Interest: The authors declare that the research was conducted in the absence of any commercial or financial relationships that could be construed as a potential conflict of interest.

Copyright (c) 2019 Nonomura and Samejima. This is an open-access article distributed under the terms of the Creative Commons Attribution License (CC BY). The use, distribution or reproduction in other forums is permitted, provided the original author(s) and the copyright owner(s) are credited and that the original publication in this journal is cited, in accordance with accepted academic practice. No use, distribution or reproduction is permitted which does not comply with these terms. 